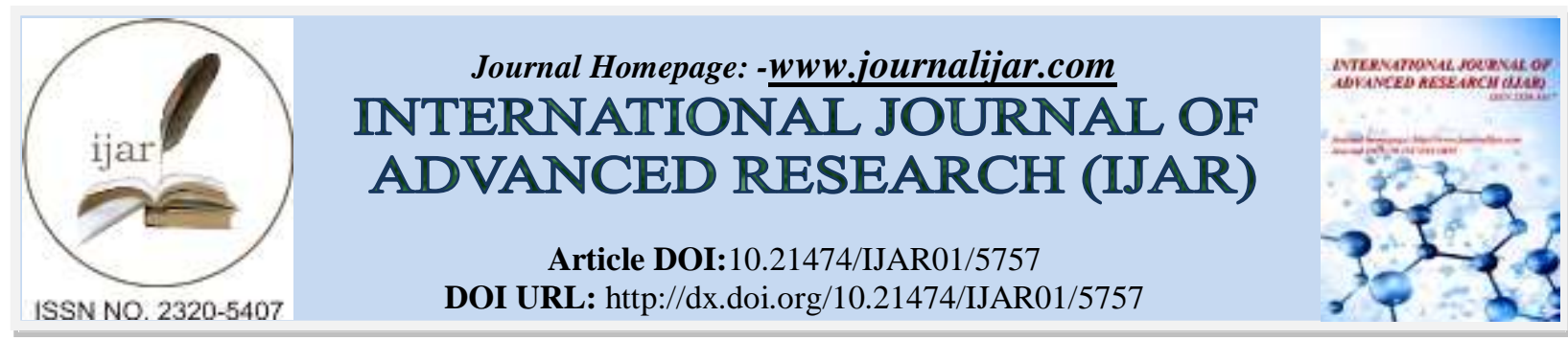

RESEARCH ARTICLE

\title{
PREVALENCE OF WORK-RELATED MUSCULOSKELETAL PROBLEMS AMONG IRONING WORKERS.
}

\author{
*A. Sankarganesh ${ }^{1}$, S. Vigneshwaran ${ }^{2}$, S. Shrisruthi ${ }^{3}$ and VPR. Siva Kumar ${ }^{4}$. \\ 1. Assistant professor, SRM College of Physiotherapy, SRM University, Chennai. \\ 2. BPT student, SRM College of Physiotherapy, SRM University, Chennai. \\ 3. BPT student, SRM College of Physiotherapy, SRM University, Chennai. \\ 4. Dean, SRM College of Physiotherapy, SRM University, Chennai.
}

\section{Manuscript Info}

Manuscript History

Received: 03 September 2017

Final Accepted: 05 October 2017

Published: November 2017

Key words:-

Ironing workers, musculoskeletal disorders, NORDIC Questionnaire.

\begin{abstract}
Background: Ironing workers are the second largest occupational group in the garment industry. These lead to the highest prevalence of repetitive traumatic and musculoskeletal disorders .The work related upper limb musculoskeletal disorders particularly consists of neck, upper extremities are the most frequently reported among them.

Objective: To find out the most common musculoskeletal disorders among ironing workers.

Methodology: Study design was Non-Experimental, study type was observational type. Inclusion criteria: both Men and Women are taken, Age 25-50.Exclusion criteria: recent fracture and surgeries. The samples are collected about 183 according to inclusion and exclusion criteria. Samples are analysed with NORDIC questionnaire and statistics are done using IBM SPSS 3620.

Results and Conclusion: Prevalence of work related musculoskeletal problems among ironing workers was predominantly increased in shoulder (69.3\%), upper back $(68.2 \%)$ and lower back (69.9\%) for last 12 months, shoulder (67.8\%), upper back $(71.6 \%)$ and lower back (71.6\%) for last 12 month prevention from work, and shoulder (67.2\%) ,upper back (72.7\%) and lower back (72.1\%) for 7 days. So we need to insist and provide them ergonomic advice which will be helpful from preventing musculoskeletal disorders.
\end{abstract}

Copy Right, IJAR, 2017,. All rights reserved.

\section{Introduction:-}

Work-related musculoskeletal disorders [WRMDs] are impairments of the bodily structures such as muscles, joints, bones, ligaments, nerves which are caused or aggravated primarily by the performance of work and by the effect of the immediate environment in which work is carried of. For the purpose of developing injury prevention strategies, many health and safety agencies include only disorders that develop gradually and are caused by the overuse of the above constituents of the musculoskeletal system. The traumatic injuries due to accidents are not considered to be WMSDs or are considered separately. However there are organisation such as European Agency for safety and health at work that include acute traumas and fractures within in the WMSD group ${ }^{1}$ 
Almost all work requires the use of arm and hands. Therefore most WMSD affect the hand, wrist, elbows, neck and shoulders. Work using the legs can lead to WMSD of the legs, hips, ankles and feet. Some back problem also result from repetitive activities.WMSDs arises from arm and hand movements such as bending, straightening, griping, holding, twisting, clenching and reaching. These common movements are not particularly harmful in the ordinary activities of daily life. The hazardous work situations are in the continuous repetition often in a forceful manner and most of all the speed of the movements and lack of time for recovery between them. WMSDs are associated with patterns that include: (1) Fixed or constrained body positions. (2) Continual repetition of movements. (3) Force concentrated on small parts of the body, such as the hand or wrist. (4) A pace of work that does not allow sufficient recovery between movements 2 .

Ironing workers are the second largest occupational group in garment industry. Ironing work is the fully manual operated and generally lacking in ergonomic design. The main action done by the ironing workers of dominant hand is more of shoulder flexion (65-75 degrees), elbow slight flexion \&extension (30-45 degrees) with elimination of gravity, wrist goes flexion(20-25 degrees), simultaneously repeated ulnar and radial, In hand MCP joints are extended, digital phalanges are completely flexed. The non-dominant hand supports the whole body swaying, the both lower limbs mainly undergoes for stress injury as they are prolong standers 2 .

At first, UCLA Southern California Education Research Centrefunded the pilot project was designed to develop and test Effectiveness of Low Cost Engineering changes to ironing work station. The research team was able to recruit a small group of ironing workers as an "ERGONOMICS COMMITTEE" who collectively evaluated and proposed changes. Ironing workers shoulders, arms and hands are in constant motion and are engaged in repeated shoulder flexion and repeated reaching and grip action ${ }^{2}$. According to recent survey $18 \%$ of ironing workers have been found in India while $26 \%$ are working as domestic workers. The main musculoskeletal problem starts due to prolonged standing and carrying a heavy weight in hand which strains the whole body. The aim of the study was to rule out the common musculoskeletal problems among ironing workers and to provide recent ergonomic changes ${ }^{3}$.

\section{Methodology:-}

The study design was non- experimental study, study type was observational, sampling method was convenient sampling, sample size was 206, while 183 are screened and included in this document, study setting was held in and around Chennai. The Subjects were selected according to inclusion and exclusion criteria. The inclusion criteria were subjects aged between 25 to 50 years, both men and women. Exclusion consists of individuals with recent injury, recent fractures, and any type of deformities. ${ }^{4}$ The whole procedure of this project was explained in detail and informed consent form was obtained .The subjects are assessed by their age, gender, posture, built, working experience, ironing machine weight, number of hour working per day, gait pattern.

Weight of the iron box, usually 5-7 kilograms, and duration of the work per day were also documented. A NORDIC QUESTIONAIRE was explained in detail and subjects were asked to fill by themselves. The questionnaire consists of three questions for evaluating trouble with the locomotive organs. The whole body is divided into nine regions. ${ }^{5}$. They areHave you at any time during last 12 month had trouble (ache, pain, discomfort, numbness) in:

Have you at any times during last 12 month been prevented from doing your work (at home or away from home) because of the trouble?

Have you had trouble at any time during the last 7 days? 

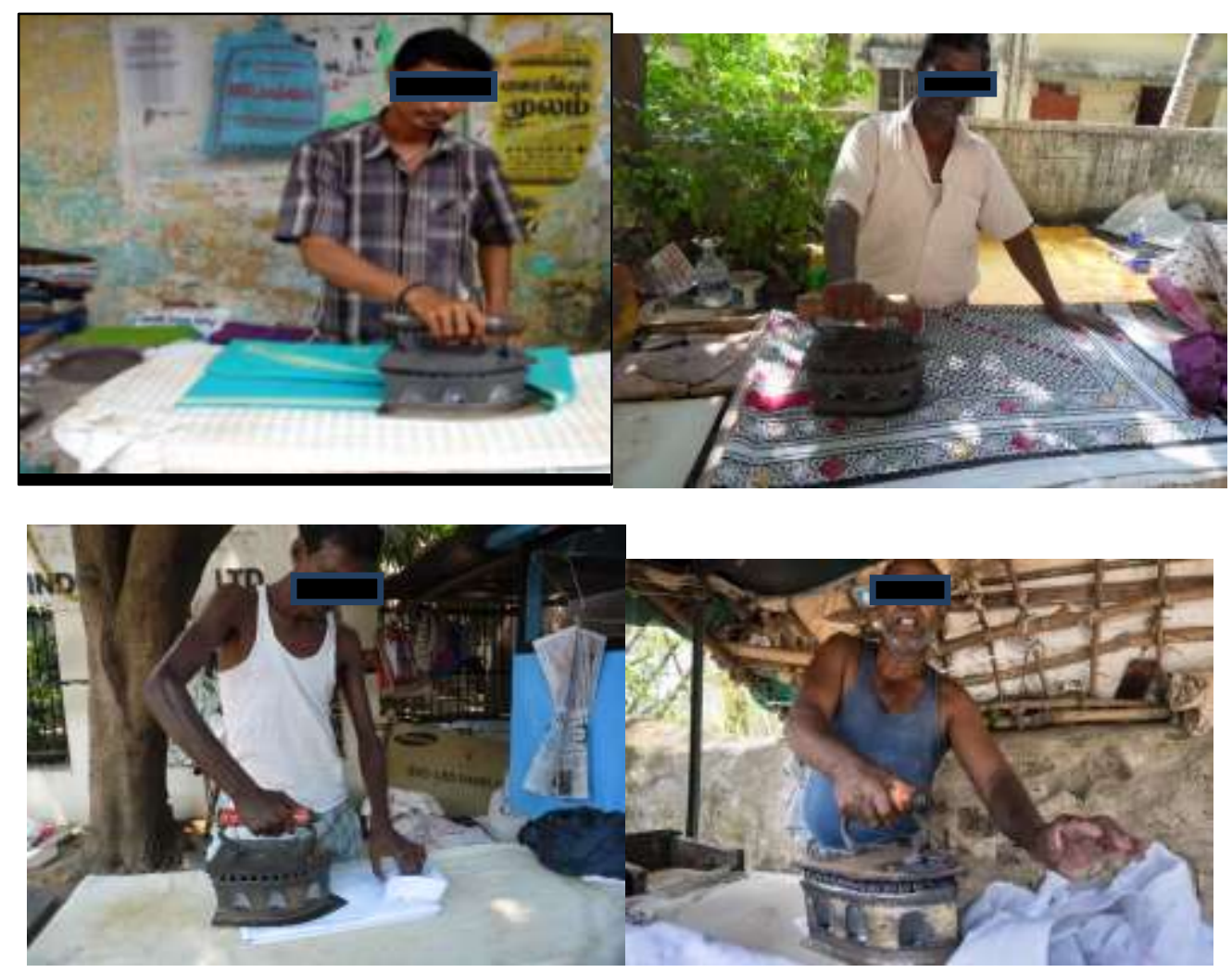

\section{Results:-}

Table 1:- Have you at any time during last 12 months had trouble (ache, pain, discomfort, numbness) in:

\begin{tabular}{|l|l|l|}
\hline Regions of pain & Positive percentage & Negative percentage \\
\hline Neck & 36.4 & 66.6 \\
\hline Shoulders & 69.3 & 30.7 \\
\hline Elbows & 55.5 & 44.5 \\
\hline Wrist/hands & 53.1 & 46.9 \\
\hline Upper back & 68.2 & 31.8 \\
\hline Lower back & 69.9 & 30.1 \\
\hline Hip/thigh & 53.2 & 46.8 \\
\hline Knee & 56.1 & 43.9 \\
\hline Ankle & 42.8 & 57.2 \\
\hline
\end{tabular}




\section{Graph 1:-}

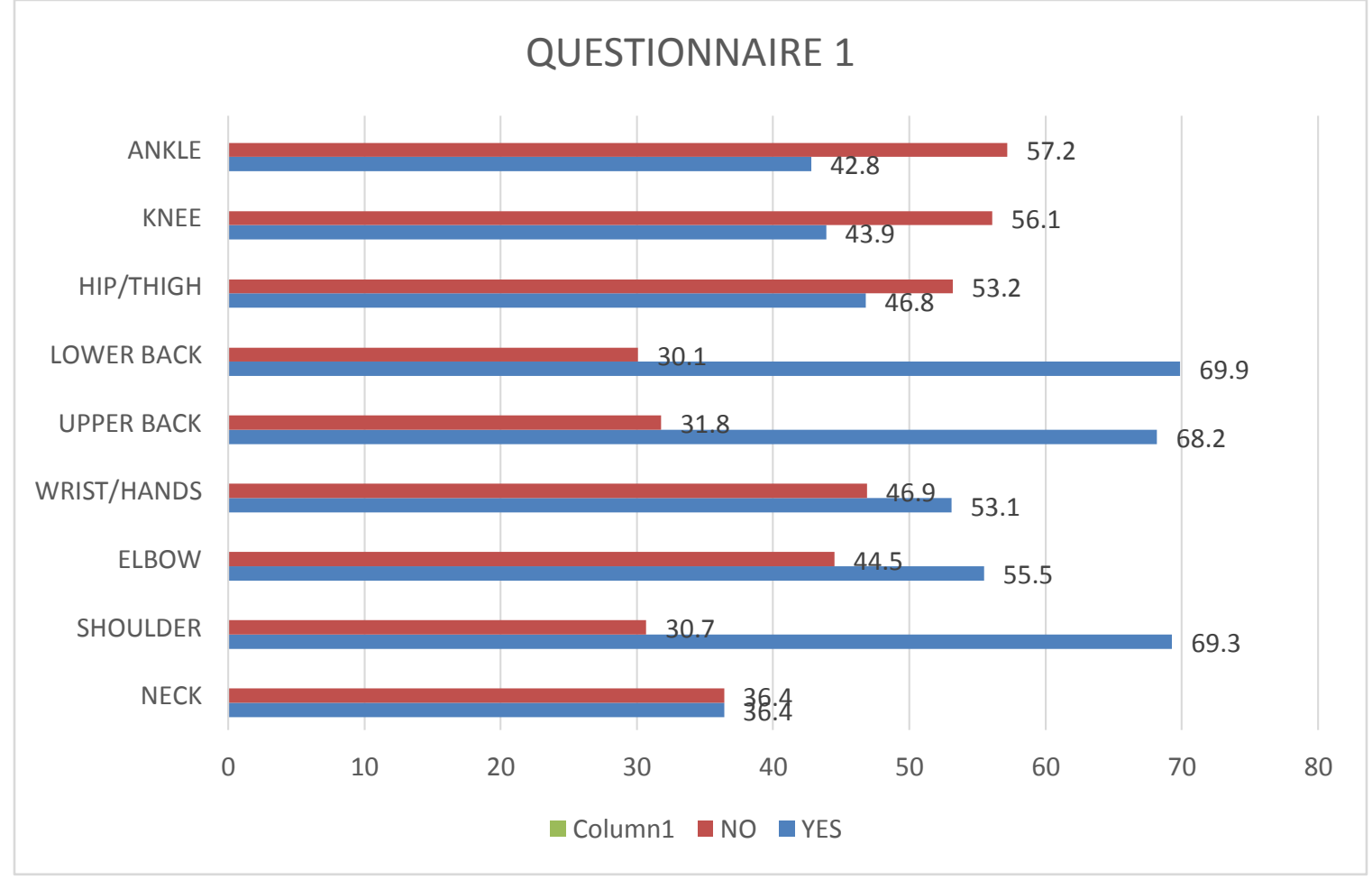

Table 1:-

From the tabulation1, the work-related musculoskeletal problems in ironing workers within 12 months with a cause of pain, ache, discomfort, numbness while working or at the rest period. The statistical analysis of any sort of pain in various regions of the body was marked from the subjects using the NORDIC Questionnaire.

Both negative and positive percentages are included in this document. According to the total subjects taken, the percentage of musculoskeletal pain is calculated as positive percentage and negative percentage. So, that the percentage can be more accurately seen in different regions. In positive percentage column the shoulders (69.3\%), lower back pain (69.9\%) are higher than other regions of the body while the neck $(36.4 \%)$ and ankle (42.8) are considered to be less. In case of negative percentage where the subjects marked with no indication of WRMDs, the highest was neck and ankle region and the least was shoulder and back region.

Table 2:- have you at any times during last 12 months been prevented from doing your work (at home or away from home) because of the trouble?

\begin{tabular}{|l|l|l|}
\hline Regions of pain & Positive percentage & Negative percentage \\
\hline Neck & 67.8 & 32.2 \\
\hline Shoulders & 67.8 & 32.2 \\
\hline Elbows & 62.3 & 37.7 \\
\hline Wrist/hands & 57.4 & 42.6 \\
\hline Upper back & 71.6 & 28.4 \\
\hline Lower back & 71.6 & 28.4 \\
\hline Hips/thigh & 68.3 & 31.7 \\
\hline Knee & 61.2 & 38.8 \\
\hline Ankle & 53.6 & 46.4 \\
\hline
\end{tabular}




\section{Graph 2:-}

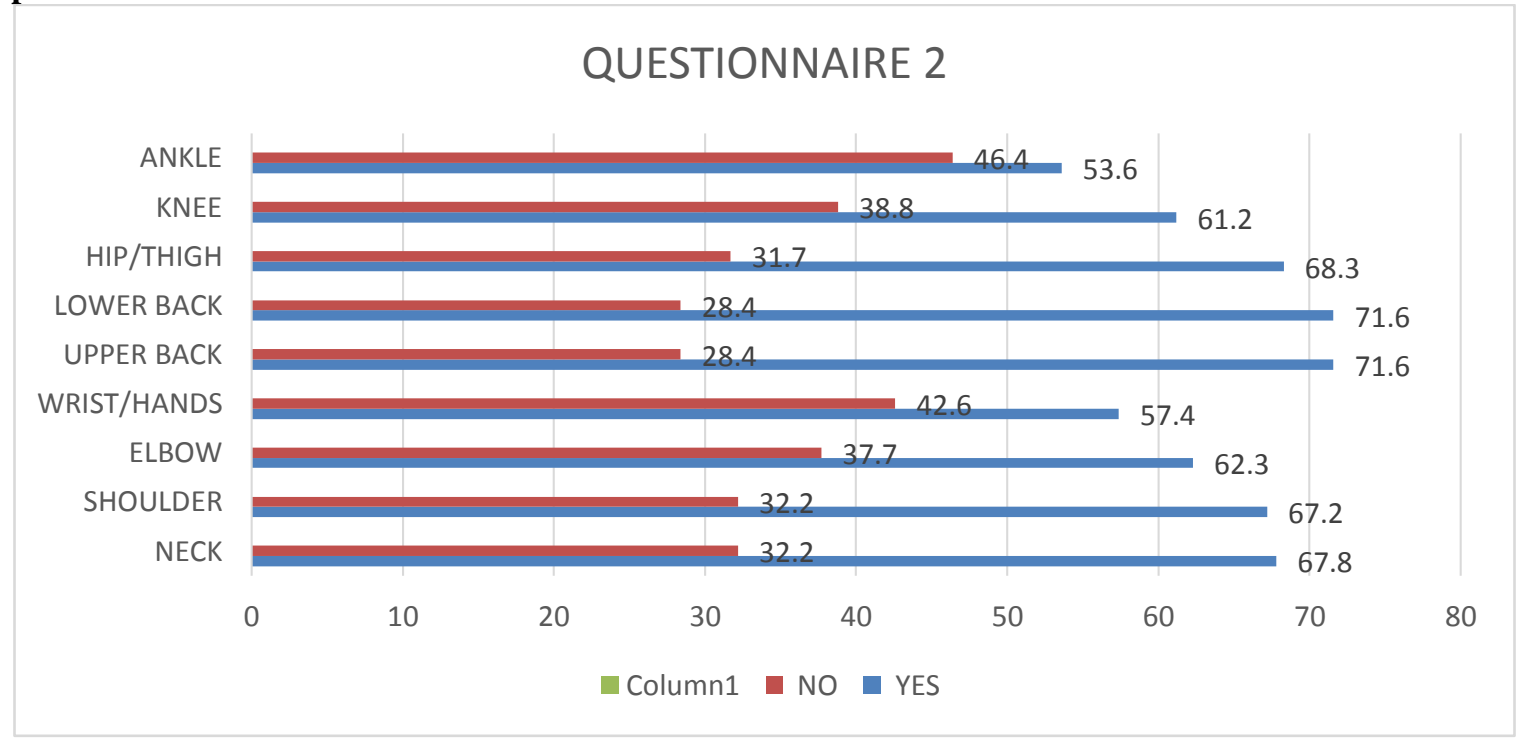

Table 2:-

In table 2 the subjects were asked to mark that they had a trouble/pain at any times during last 12 months for all the 9 regions for both the positive and negative percentage have been done, In positive percentage the neck, shoulders, hips/thigh, upper back and lower back region have highest percentage and ankle and wrist have lesser percentage. In positive percentage column the upper back (71.6\%), lower back pain (71.6\%) ,hips/thigh (68.3) neck (67.8), shoulders (67.8) are higher than other regions of the body, while the wrist/hand (42.6)\%) and ankle (48.4\%) are considered to be less.

Table 3:- have you had trouble at any time during the last 7 days?

\begin{tabular}{|l|l|l|}
\hline Regions of pain & Positive percentage & Negative percentage \\
\hline Neck & 67.8 & 32.2 \\
\hline Shoulders & 67.2 & 32.8 \\
\hline Elbows & 62.3 & 37.7 \\
\hline Wrist/hands & 57.4 & 42.6 \\
\hline Upper back & 72.7 & 27.3 \\
\hline Lower back & 72.1 & 27.9 \\
\hline Hips/thigh & 65 & 35 \\
\hline Knee & 60.1 & 39.9 \\
\hline Ankle & 50.8 & 49.2 \\
\hline
\end{tabular}




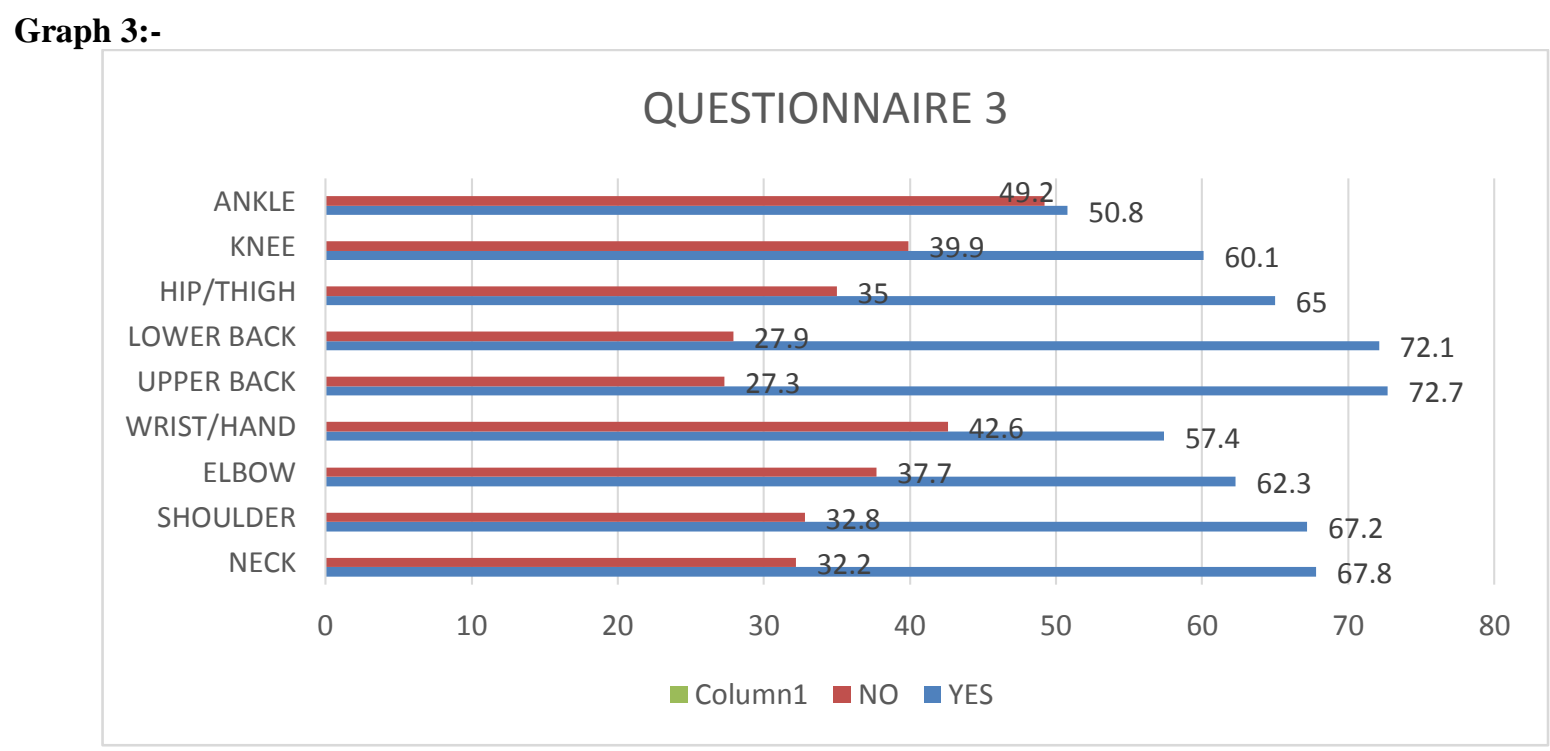

Table 3:-

In table 3, the subjects were asked to mark that they had a trouble/pain at any times during last 7 days for all the 9 regions for both the positive and negative percentage have been done. In positive percentage the upper back and shoulder region have highest percentage and wrist/hands have lesser percentage, but in negative percentage the highest is ankle and least is upper back region. Comparative to the other this table shows the massive difference in between negative and positive percentage.

\section{Discussion:-}

The aim of the study was to find out the prevalence of musculoskeletal problems among ironing workers. From the above data it has been proved that the musculoskeletal pain is the variantly seen in ironing workers. The statistical analysis of IBM SPSS 3620 version shows that the pain in mainly seen in shoulders, upper and lower back and the regions with less variant is ankle joint of both legs. The data have been collected for various regions of the body such as Neck, Shoulder-right, left, both sides have been included, Elbow-right, left, both sides are included. Upper back, lower back, Hip/Thigh region-both sides, Knee joint-both sides, Ankle joint-both side ${ }^{2}$.

Due to the heavy work load over the shoulders which leads to biomechanical postural changes over the whole body especially certain regions such as shoulders undergoes static and dynamic scapula humeral rhythm, soft tissue injuries such as supraspinatus tendinitis, bicipital tendinitis and lumbo pelvic rhythm, gait patterns can be seen. These musculoskeletal problems lead to have a poor quality of life among them. These problems are more prone in dominant side users than non-dominant side few such as spasm, swelling, inflammation are predominantly seen early in dominant side and the study shows that the subjects have radiating pain from dominant to non-dominant side both in upper limb as well as lower limb. Not only the upper limb and lower limb, the trunk also undergoes for biomechanical changes such as nerve root impingement, compression causes change in dermatome and myotome. The hand grip strength of the ironing workers can be examined in further studies. The limitations and recommendations are larger sample size, Ergonomic work station design can be implemented, and Quality of life should be checked. Pulmonary parameters can be assisted in further studies .Postural change examination and correction is indeed. Age and gender wise statistical analysis can be done.

\section{Conclusion:-}

This study concludes that prevalence of work related musculoskeletal problems among ironing workers was predominantly increased in shoulder $(69.3 \%)$, upper back $(68.2 \%)$ and lower back $(69.9 \%)$ for last 12 months, shoulder $(67.8 \%)$, upper back $(71.6 \%)$ and lower back $(71.6 \%)$ for last 12 month prevention from work, and shoulder $(67.2 \%)$, upper back $(72.7 \%)$ and lower back $(72.1 \%)$ for 7 days. There are variant changes in ergonomics posture, and they are unaware of further complications. So we need to insist and provide them ergonomic advice which will be helpful from preventing musculoskeletal disorders. 


\section{References:-}

1. Woolf, A D and akesson, $\mathrm{K}, 2001$, Understanding the burden of musculoskeletal conditions. The burden is huge and not reflected in national health priorities. BMJ ,322: 1079_1080.

2. Hagberg. M. 1995. “Conceptual framework". In work Related Musculoskeletal Disorder (WMSDs): A Reference Book for Prevention, Edited by:Kuorinka ,I Forcier,L.5-16. London: Taylor \&Francis. WMSDs.

3. Buckle P. Ergonomics and musculoskeletal disorders: overview. Occup Med (Lond) (2005:55:164 -7).

4. Anderson, K, Karlehagen , S and Jonsson , B 1987. The importance of variations in questionnaire administration. ApplErgon, $18: 229$-32.

5. Kuorinka, I, Jonsson, BKilbom , A.1987 Standardised Nordic Questionaires for the analysis for musculoskeletal symptoms. ApplErgon, $18: 233$-237.

6. OhlssonK,AttewellR,PaissonB,KarlssonB,Balogh I, Johnsson B, et al.Repetitive industrial work and neck and upper limb disorders in females. Am J Ind Med.1995;27:731-47.

7. Eckardt J .Evaluation and management of occupational low back disorders. Am J Med.2000:37:94-111.

8. Putz-Anderson V. Cumulative trauma disorders-a manual for musculoskeletal diseases of the upper limbs. London, UK: Taylor \&Francis: 1988.

9. Hagberg M, Wegman DH. Prevalence rates and odds ratios of shoulder-neck disease in different occupational groups.Br J Ind Med.1987;44:602-10.

10. Buckwalter JA, Woo SY, Goldberg VM, Hadley EC, Booth F, oegema TR, et al. Soft - tissue aging and musculoskeletal function. J Bone joint surg am.1993:1533-48.

11. Stock SR, Workplace ergonomics factors and the development of musculoskeletal disorders of the neck and upper limbs: a meta - analysis. Am J Ind med. 1991.

12. Dempsey PG, Burdort A, Webster BS. The influence of personl variable on work related low back disorders and implication for future research.Joccup Environ Med. 1997:39:748-59. 